GEOLOGICAL SURVEY

\title{
Analytical results and sample locality maps of rock samples from the northwestern Idaho Falls and the northeastern \\ Hailey 1 × 2 degree quadrangles, Idaho
}

By

John H. Bullock Jr., ${ }^{\star}$ Anna B. Wilson, ${ }^{\star \star}$ and

Sandra J. Soulliere**

Open-File Report 90-645

This report is preliminary and has not been reviewed for conformity with U.S.

Geological Survey editorial standards. Any use of trade, product or firm names is for descriptive purposes only and does not imply endorsement by the USGS.

*U.S. Geological Survey, DFC, Box 25046, MS 973, Denver, CO 80225

**U.S. Geological Survey, DFC, Box 25046, MS 905, Denver, CO 80225 


\section{CONTENTS}

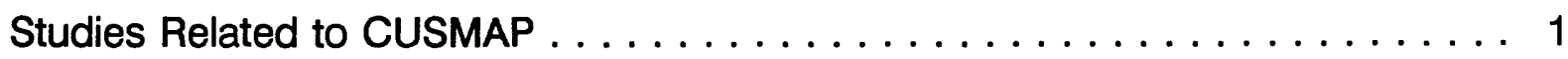

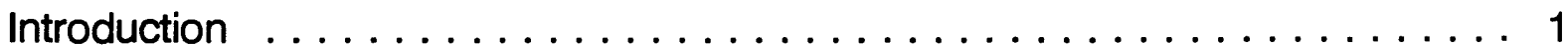

Methods of Study $\ldots \ldots \ldots \ldots \ldots \ldots \ldots \ldots \ldots \ldots \ldots \ldots$

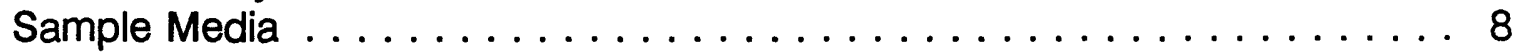

Sample Collection and Preparation $\ldots \ldots \ldots \ldots \ldots \ldots \ldots \ldots$

Sample Analysis $\ldots \ldots \ldots \ldots \ldots \ldots \ldots \ldots \ldots \ldots \ldots$

Data Storage System . . . . . . . . . . . . . . . . . . . . 8

Description of Data Tables . . . . . . . . . . . . . . . . . 9

References Cited ......................... 9

\section{ILLUSTRATIONS}

Figure 1. Location of the northwestern Idaho Falls and northeastern Hailey

$1 \times 2$ degree quadrangles, including mining districts $\ldots \ldots \ldots \ldots 2$

Figure 2. Localities of rock samples from the Mackay 15-minute quadrangle . . . 3

Figure 3. Localities of rock samples from the Copper Basin 15-minute quadrangle ........................ 4

Figure 4. Localities of rock samples from the Muldoon Canyon 15-minute

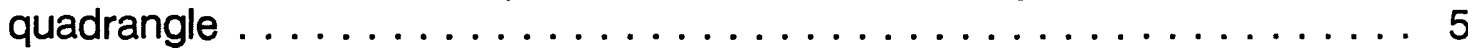

Figure 5. Localities of rock samples from the Grouse 15-minute quadrangle . . 6

Figure 6. Localities of rock samples from the Phi Kappa Mountain 7.5-minute quadrangle $\ldots \ldots \ldots \ldots \ldots \ldots \ldots \ldots \ldots \ldots \ldots \ldots \ldots \ldots \ldots \ldots$

\section{TABLES}

Table 1. Sample descriptions $\ldots . . \ldots \ldots \ldots \ldots \ldots \ldots . \ldots \ldots 11$

Table 2. Limits of determination for spectrographic analysis of rock samples . . 14

Table 3. Results of analyses . . . . . . . . . . . . . . . . . 15 


\section{STUDIES RELATED TO CUSMAP}

This report presents the results of a partial geochemical survey of a part of the Idaho Falls and the Hailey $1 \times 2$ degree quadrangles, Idaho. Samples for geochemical analyses were collected as one of several multidisciplinary studies associated with a U.S. Geological Survey Conterminous United States Mineral Appraisal Program (CUSMAP) to evaluate the mineral resources of the Hailey $1 \times 2$ degree quadrangle and part of the Idaho Falls $1 \times 2$ degree quadrangle, Idaho.

\section{INTRODUCTION}

In 1987, the U.S. Geological Survey conducted a reconnaissance geochemical survey of rock-chip and stream-sediment samples from the northwestern Idaho Falls $1 \times 2$ degree quadrangle and the northeastern part of the Hailey $1 \times 2$ degree quadrangle, Idaho (fig. 1). Sixty-five rock-chip samples and two stream-sediment samples were collected from five areas within the Idaho Falls and the Hailey $1 \times 2$ degree quadrangles: Mackay, Copper Basin, Muldoon Canyon, and Grouse 15-minute quadrangles, and Phi Kappa Mountain 7.5-minute quadrangles (figs. 2-6). This report presents the results of the geochemical analyses.

Geochemical sampling was concentrated on known mineral deposits from five mining districts: Alder Creek, Copper Basin, Little Wood River, Lava Creek, and Alto (fig. 1). Three types of mineral deposits have been exploited intermittently from these districts since their discovery in the late 1800's: skarn deposits, polymetallic veins in Paleozoic sedimentary rocks, and polymetallic veins in volcanic rocks (Worl and others, 1989). These deposits produced copper, lead, zinc, silver, gold, tungsten, molybdenum, barite, iron, and fluorspar (Nelson and Ross, 1969a). Current exploration in the region is focused on disseminated gold deposits in volcanic and sedimentary terranes and on gold skarns. There does not appear to be an interest in base metals at the present time.

Geology was previously mapped and described by Nelson and Ross (1968, 1969a and b), Skipp (1988, 1989) and Skipp and others (1990). Geologic units are Paleozoic rocks, mainly carbonates and clastics, intruded by Tertiary granitic stocks and hypabyssal bodies and overlain by Eocene volcanic rocks. Several north- and northeast-striking faults cut all rock types. A more complete discussion of these strata and their structural relations is discussed in Link and others (1988) and Worl and others (1989). 


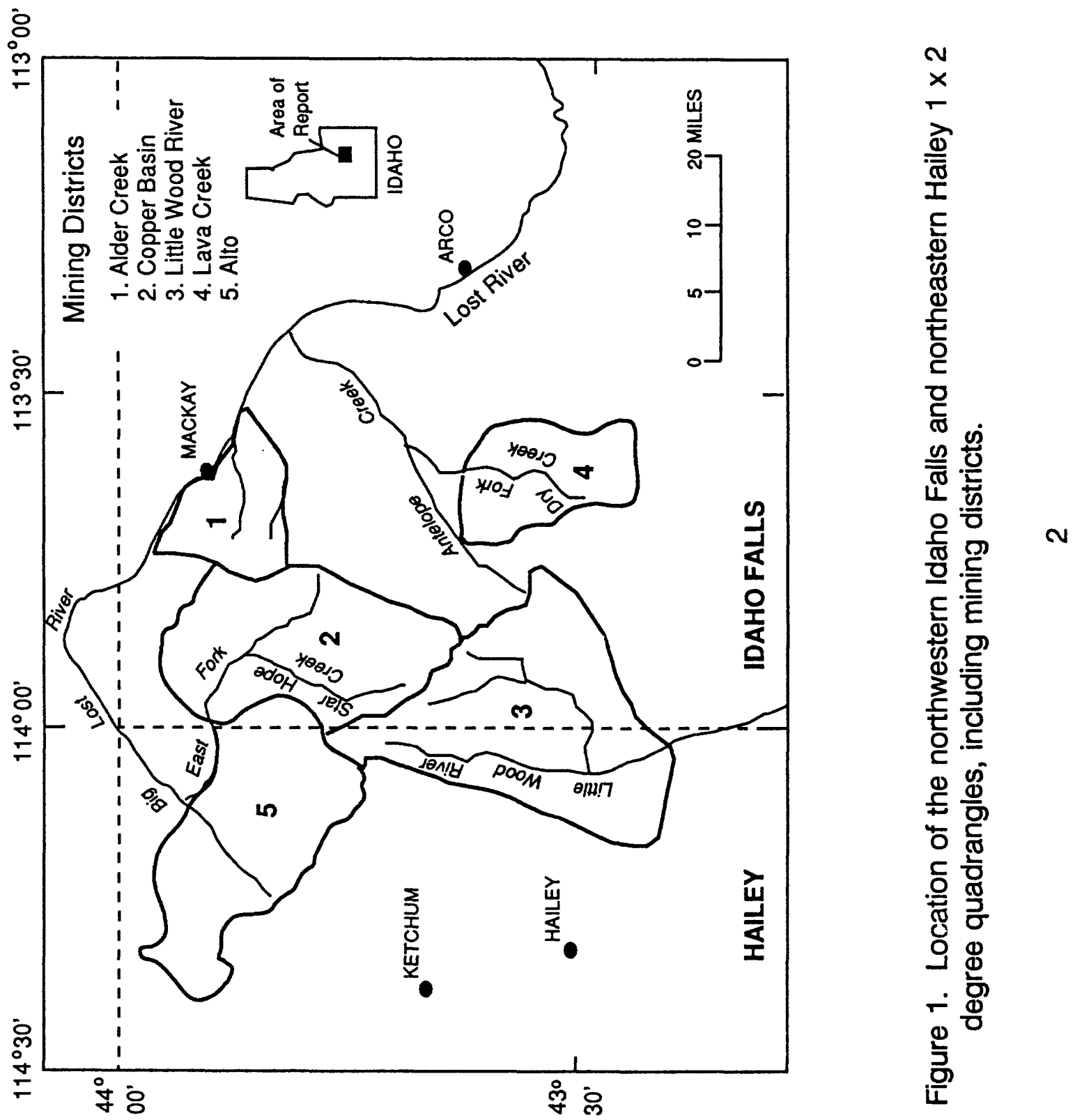




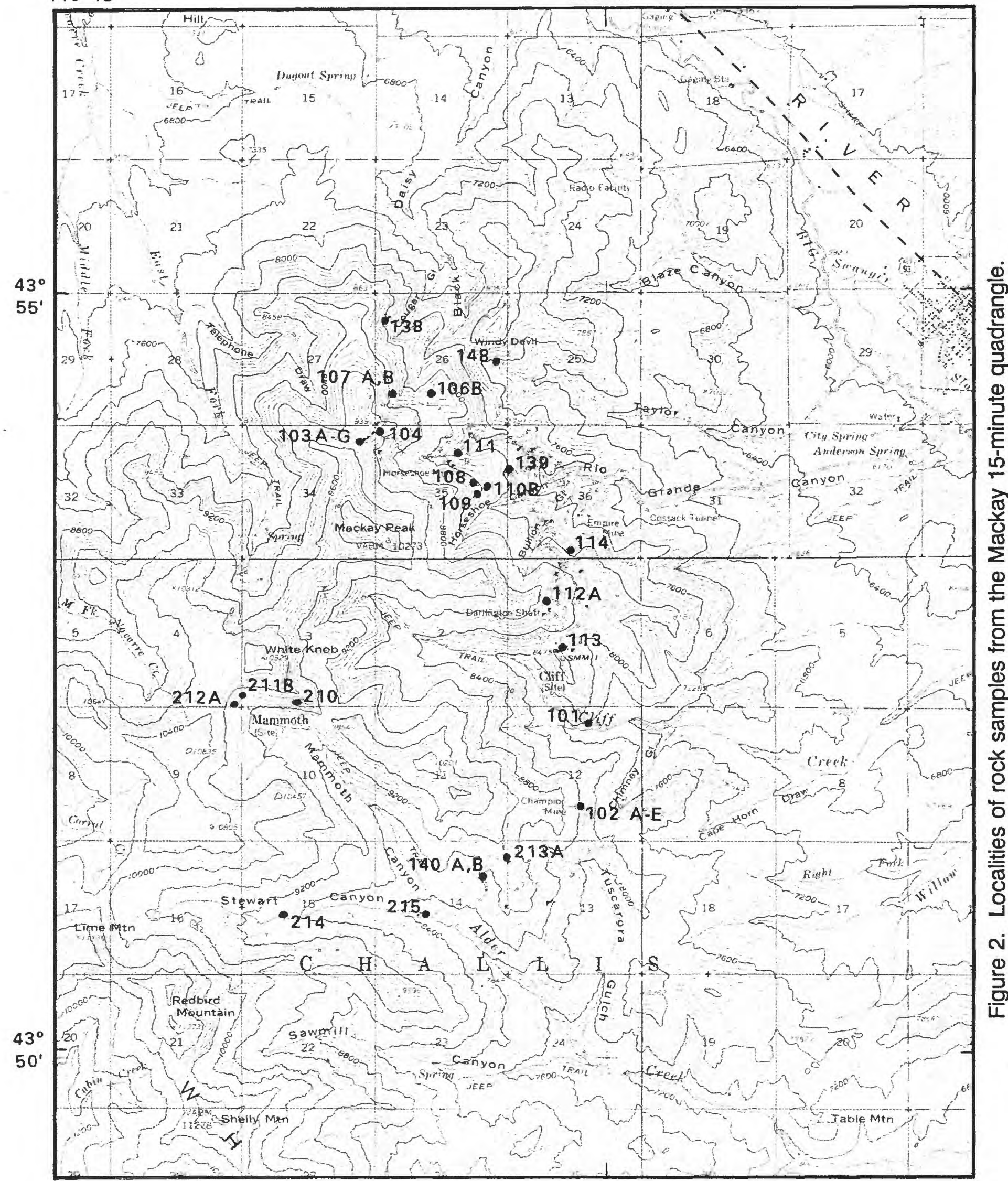

Base from the USGS; $1: 62,500$

Mackay, Idaho, 1960

$1 / 2$

3

4 MILES 


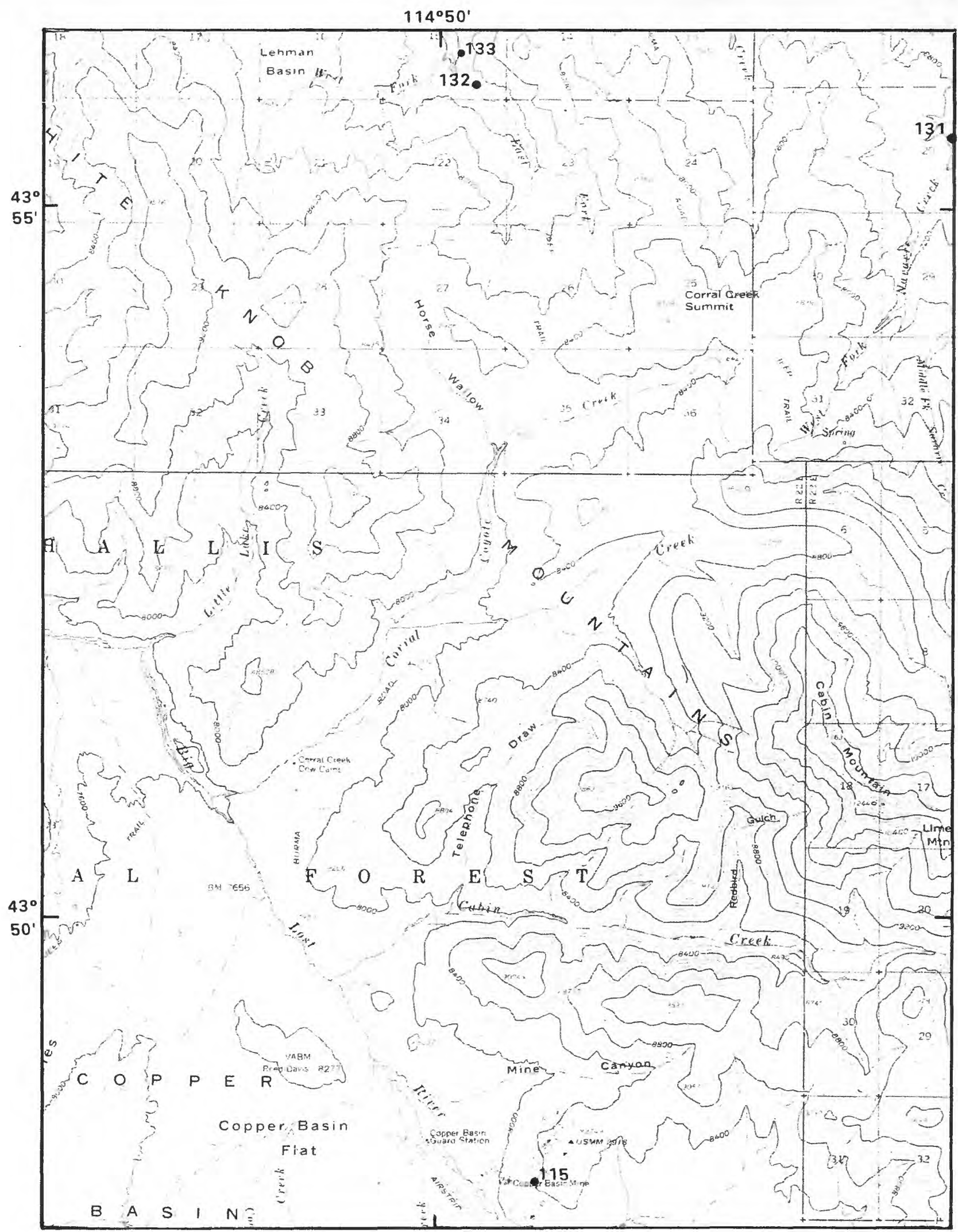

Base from the USGS;

Copper Basin, Idaho, 1960

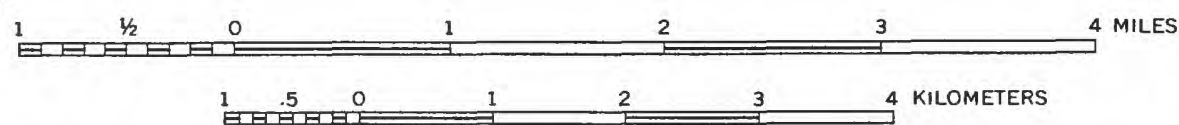

Figure 3. Localities of rock samples from the Copper Basin 15-minute quadrangle. 


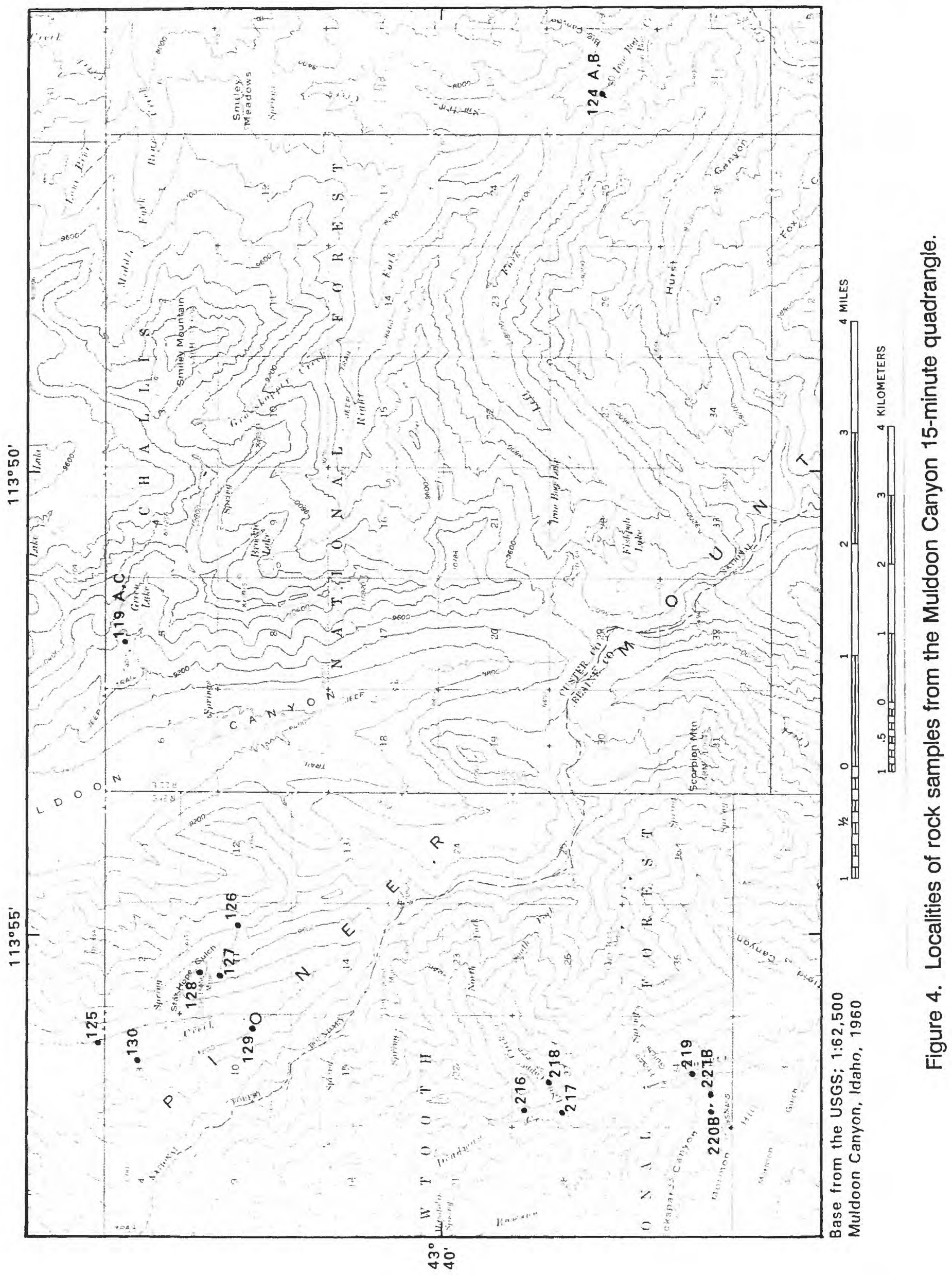




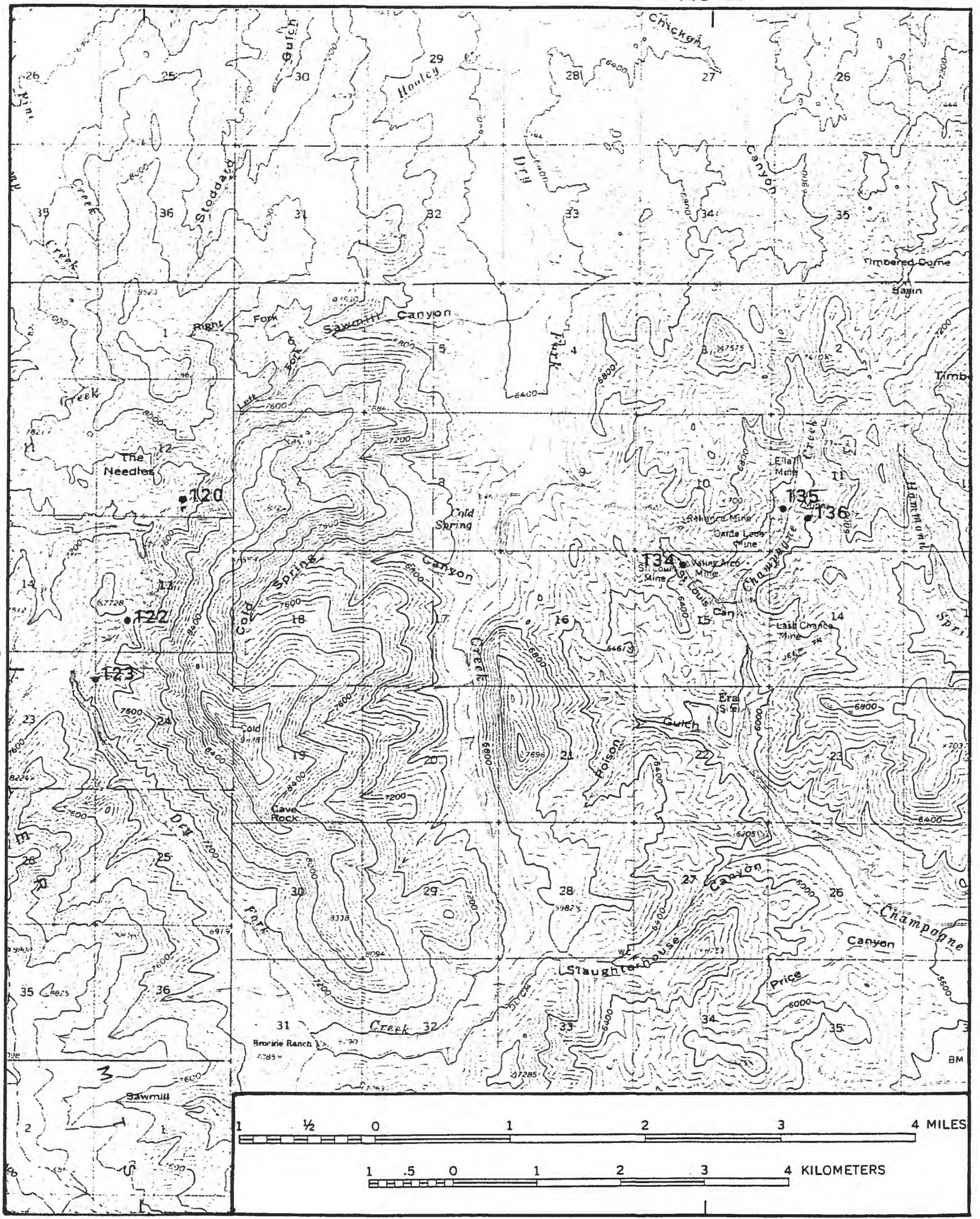

Base from the USGS; $1: 62,500$

Grouse, Idaho, 1960

Figure 5. Localities of rock samples from the Grouse 15-minute quadrangle. 


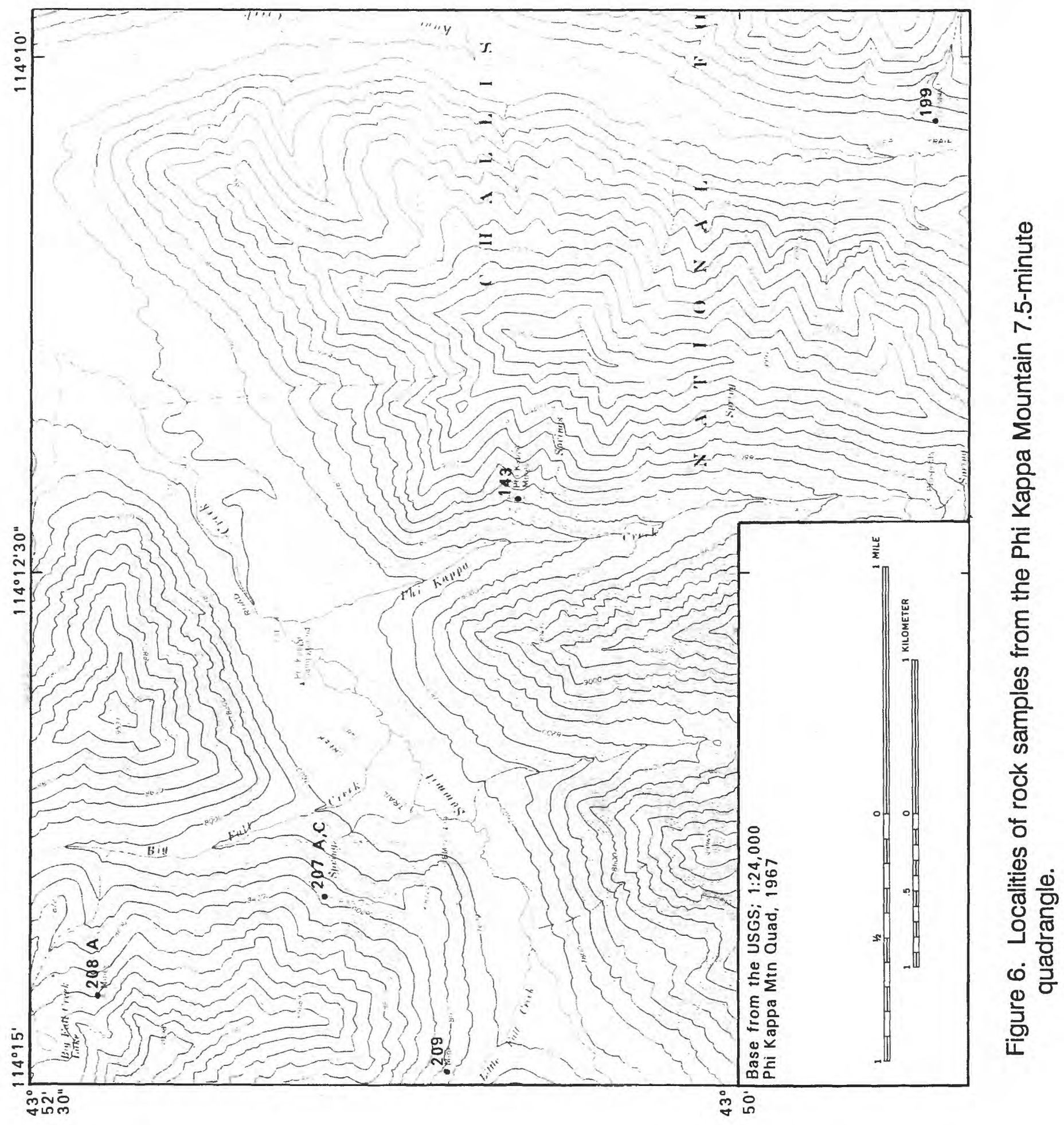




\section{METHODS OF STUDY}

\section{Sample Media}

Analyses of unmineralized or unaltered rock samples provide background geochemical data for individual rock units. Analyses of mineralized or altered rocks may provide useful goechemical information about the major and trace-element assemblages associated with a possible mineralizing system.

\section{Sample Collection and Preparation}

Samples were collected from 65 sites (figs. 2-6). At each site, a 5-lb bag of rock chips and one or more hand samples were collected. In addition, two streamsediment samples were collected from the Stewert Canyon drainage. Sample descriptions are located in table 1.

The samples were crushed and then pulverized to approximately minus-100 mesh (minus- $0.15 \mathrm{~mm}$ ) with ceramic plates.

\section{Sample Analysis}

All samples were analyzed for 35 elements using a semiquantitative, direct-current arc emission spectrographic method (Grimes and Marranzino, 1968). The elements analyzed and their limits of determination are listed in table 2.

Spectrographic results were obtained by visual comparison of spectra derived from the sample against spectra obtained from standards made from pure oxides and carbonates. Standard concentrations are geometrically spaced over any given order of magnitude of concentration as follows: 100,50,20,10, and so forth. Samples whose concentrations are estimated to fall between those values are assigned values of $70,30,15$, and so forth. The precision of the analytical method is approximately plus or minus one reporting interval at the 83 percent confidence level and plus or minus two reporting intervals at the 96 percent confidence level (Motooka and Grimes, 1976). Values determined for the major elements (iron, magnesium, calcium, phosphorus, sodium, and titanium) are given in weight percent; all others are given in parts per million (micrograms/gram). Analytical results are listed in table 3.

\section{DATA STORAGE SYSTEM}

Upon completion of the analytical work, the results were entered into a U.S. Geological Survey computer data base called PLUTO. This data base contains both descriptive geological information and analytical data. Any or all of this information may be retrieved and converted to a binary form (STATPAC) for computerized statistical analysis or publication (VanTrump and Miesch, 1977). 


\section{DESCRIPTION OF DATA TABLES}

Table 3 lists the results of analyses of rock samples from the study area. The data are arranged so that column 1 contains the sample field numbers. These numbers correspond to the numbers shown on the site location maps (figs. 2-6). The letter "s" underneath the column headings indicates emission spectrographic analyses. A letter " $\mathrm{N}$ " in the tables indicates that a given element was looked for but not detected at the lower limit of determination shown for that element in table 2. A "less than" symbol $(<)$ entered in the tables in front of the lower limit of determination indicates that the element was observed but was below the lowest reporting value. If an element was observed but was above the highest reporting value, a "greater than" symbol (>) was entered in front of the upper limit of determination. Because of the formatting used in the computer program that produced table 3, some of the elements listed in these tables ( $\mathrm{Fe}, \mathrm{Mg}, \mathrm{Ca}, \mathrm{Ti}, \mathrm{Ag}$, and $\mathrm{Be}$ ) may carry one or more nonsignificant digits to the right of the significant digits. The analyst did not determine these elements to the accuracy suggested by the extra zeros.

\section{REFERENCES CITED}

Grimes, D.J., and Marranzino, A.P., 1968, Direct-current arc and alternating-current spark emission spectrographic field methods for the semiquantitative analysis of geologic materials: U.S. Geological Survey Circular 591, 6 p.

Link, P.K., Skipp, Betty, Haitt, M.H., Jr., Janecke, Suzanne, and Burton, B.R., 1988, Structural and stratigraphic transect of south-central Idaho--A field guide to the Lost River, White Knob, Pioneer, Boulder, and Smoky Mountains, in Link, P.K., and Hackett, W.R. eds., Guidebook to the geology of central and southern Idaho: Idaho Geological Survey Bulletin 27, p. 5-42.

Motooka, J.M., and Grimes, D.J., 1976, Analytical precision of one-sixth order semiquantitative spectrographic analyses: U.S. Geological Survey Circular 738, $25 \mathrm{p}$.

Nelson, W.H., and Ross, C.P., 1968, Geology of part of the Alder Creek mining district, Custer County, Idaho: U.S. Geological Survey Bulletin 1252-a, 30 p.

Nelson, W.H., and Ross, C.P. 1969a, Geology of the Mackay 30-minute quadrangle, Idaho: U.S. Geological Survey Open-File Report 1385, 161 p.

Nelson, W.H., and Ross, C.P., 1969b, Geologic map of the Mackay quadrangle, southcentral, Idaho: U.S. Geological Survey Miscellaneous Geologic Investigations Map I-580, scale 1:125,000.

Skipp, Betty, 1988, Geologic map of Mackay 4 (Grouse) NE quadrangle, Butte and Custer Counties, Idaho: U.S. Geological Survey Open-File Report 88-423, scale $1: 24,000$.

Skipp, Betty, 1989, Geologic map of Mackay 4 (Grouse) NW quadrangle, Butte and Custer Counties, Idaho: U.S. Geological Survey Open-File Report 89-142, scale $1: 24,000$. 
Skipp, Betty, Kuntz, M.A. and Morgan, L.A., 1990, Geologic map of Mackay 4 (Grouse) SE quadrangle, Butte County, Idaho: U.S.Geological Survey Open-File Report 89-431, scale 1:24,000.

VanTrump, George, Jr., and Miesch, A.T., 1977, The U.S. Geological Survey RASSSTATPAC system for management and statistical reduction of geochemical data: Computers and Geosciences, v. 3, p. 475-488.

Worl, R.G., Wilson, A.B., Smith, C.L., Kleinkopf, M.D., and Sykes, R.C., 1989, Mineral resource potential and geology of the Challis National Forest, Idaho: U.S. Geological Survey Bulletin 1873, 101 p. 
Table 1. Sample Descriptions

Sample Description

MACKAY QUADRANGLE (35)

87M-101 Cliff Creek adit, dump sample

87M-102A Champion mine, gray limestone, outcrop sample

87M-102B Champion mine, gray shale, noncalcareous, outcrop sample

87M-102C Champion mine, calc-silicate, outcrop sample

87M-102D Champion mine, dump sample

$87 \mathrm{M}-102 \mathrm{E}$

Champion mine, breccia, outcrop sample

87M-103A

87M-103B

$87 \mathrm{M}-103 \mathrm{C}$

Tip Top claims, outcrop sample, granodiorite

Tip Top claims, outcrop sample, altered granodiorite

Tip Top claims, outcrop sample, altered White Knob Limestone

87M-103D Tip Top claims, dump sample

87M-103E Tip Top claims, outcrop sample, White Knob Limestone

87M-103F Tip Top claims, outcrop sample, calc-silicate skarn

87M-103G Tip Top claims, outcrop sample, recrystallized White Knob Limestone

87M-104 Tip Top claims, dump sample with visible galena

87M-106B Nicor claims, grab sample from waste pile

87M-107A Unnamed adits at head of Black Daisy Canyon, rock chip sample from adit, dike rock

87M-107B

Horseshoe mine area, dump sample

$87 \mathrm{M}-108$

$87 \mathrm{M}-109$

87M-110B

87M-111

87M-112A

87M-113

87M-114

87M-138

87M-139

87M-140A

$87 \mathrm{M}-140 \mathrm{~B}$

87M-148

87M-210

87M-211B

87M-212A

87M-213A

Horseshoe mine area, rock-chip sample across face of unnamed adit

Horseshoe mine area, dump sample, copper oxide staining

Horseshoe mine area, dump sample, visible pyrite and galena, sulphurus aroma

Empire mine, open pit, granodiorite

Grand Prize mine, dump sample

Empire mine, Alberta Tunnel, dump sample

Veteran mine, dump sample

Bluebird mine, dump sample

Lower Harryaune prospect, dump sample

Lower Harryaune prospect, rock chips across shear

zone in adit, limestone

Black Queen claims, dump sample

Mammoth mine, dump sample

Silver Lead Bell group, dump sample

Do

Upper Harryaune prospect, dump sample 
Table 1. Sample Descriptions--Continued

Sample Description

87M-214 Stewart Canyon stream-sediment sample

$87 \mathrm{M}-215$ Do

COPPER BASIN QUADRANGLE (4)

87M-115 Copperhead claims, trench wall sample

87M-131 Navarre Creek prospects, dump sample

87M-132 Upper Lehman Creek adit, dump sample

87M-133 Lower Lehman Creek adit, grab sample

MULDOON CANYON QUADRANGLE (16)

87M-119A Candy Cane group, vein sample from adit

87M-119C Candy Cane group, black shale on dump

87M-124A Iron Bog mine, black shale and conglomerate

87M-124B Iron Bog mine,limonite stained conglomerate

87M-125 Adit along Star Hope Creek, dump sample

87M-126 Plumbo claims, ridge at head of Star Hope Gulch, black shale, Copper Basin Formation

87M-127 Star Hope mine, upper adit, dump sample, visible copper oxides

87M-128 Star Hope mine, lower adit, dump sample, visible galena, quartzite

87M-129 Bent Pine Tree group, dump sample

87M-130 Mackinaw group, dump sample, chalcopyrite, galina in calc-silicate

87M-216 Deadman Creek, outcrop sample, quartzite with visible pyrite

87M-217 Caved adit, dump sample

87M-218 Adit along Little Copper Creek, outcrop sample across shear zone in black shale

87M-219 Blackspar mine, dump sample

$87 \mathrm{M}-220 \mathrm{~B}$

87M-221B

Do

Do

GROUSE QUADRANGLE (6)

87M-120 Leadbelt mine, dump sample

87M-122 Outcrop sample, McGowan Creek Formation

87M-123 Caved adit south of Leadbelt mine, dump sample

87M-134 St. Louis mine, dump sample

87M-135 Ella mine, dump sample

87M-136 Honsilver mine, silicified Challis Volcanics 
Table 1. Sample Descriptions--Continued

Sample Description

PHI KAPPA MTN QUADRANGLE (6)

87M-143 Phi Kappa mine, dump sample

87M-199 Kane Creek prospect pit sample

87M-207A White Elephant mine, adit wall sample

87M-207C White Elephant mine, prospect pit samples

87M-208A Black Rock claims, dump sample

87M-209 Little Falls Creek prospect, dump sample 

2
TABLE 1.--Limits of determination for the spectrographic analys is of
rocks, based on a $10-\mathrm{mg}$ sample

\begin{tabular}{lcc}
\hline Elements & Lower determination limit & Upper determination limit \\
\hline & Weight percent & \\
\hline Calcium ( $\mathrm{Ca})$ & 0.05 & 20 \\
Iron ( $\mathrm{Fe})$ & .05 & 20 \\
Magnesium (Mg) & .02 & 10 \\
Sodium ( $\mathrm{Na}$ ) & .2 & 5 \\
Phosphorus (P) & .2 & 10 \\
Titanium (Ti) & .002 & 1 \\
\hline
\end{tabular}

Parts per million

\begin{tabular}{|c|c|c|}
\hline $\begin{array}{l}\text { Silver (Ag) } \\
\text { Arsenic (As) } \\
\text { Gold (Au) } \\
\text { Boron (B) } \\
\text { Barium (Ba) } \\
\text { Beryllium (Be) } \\
\text { Bismuth (Bi) } \\
\text { Cadmium (Cd) } \\
\text { Cobalt (Co) } \\
\text { Chromium (Cr) } \\
\text { Copper (Cu) } \\
\text { Gallium (Ga) } \\
\text { Germanium (Ge) } \\
\text { Lanthanum (La) } \\
\text { Manganese (Mn) } \\
\text { Molybdenum (Mo) } \\
\text { Niobium (Nb) } \\
\text { Nickel (Ni) } \\
\text { Lead (Pb) } \\
\text { Antimony (SD) } \\
\text { Scandium (SC) } \\
\text { Tin (Sn) } \\
\text { Strontium (Sr) } \\
\text { Thorium (Th) } \\
\text { Vanadium (V) } \\
\text { Tungsten (W) } \\
\text { Yttrium (Y) } \\
\text { Zinc (Zn) } \\
\text { Zirconium (Zr) }\end{array}$ & $\begin{array}{c}0.5 \\
200 \\
10 \\
10 \\
20 \\
1 \\
10 \\
20 \\
10 \\
10 \\
5 \\
5 \\
10 \\
50 \\
10 \\
5 \\
20 \\
5 \\
10 \\
100 \\
5 \\
10 \\
100 \\
100 \\
10 \\
20 \\
10 \\
200 \\
10\end{array}$ & $\begin{array}{r}5,000 \\
10,000 \\
500 \\
2,000 \\
5,000 \\
1,000 \\
1,000 \\
500 \\
2,000 \\
5,000 \\
20,000 \\
500 \\
100 \\
1,000 \\
5,000 \\
2,000 \\
2,000 \\
5,000 \\
20,000 \\
10,000 \\
100 \\
1,000 \\
5,000 \\
2,000 \\
10,000 \\
10,000 \\
2,000 \\
10,000 \\
1,000\end{array}$ \\
\hline
\end{tabular}




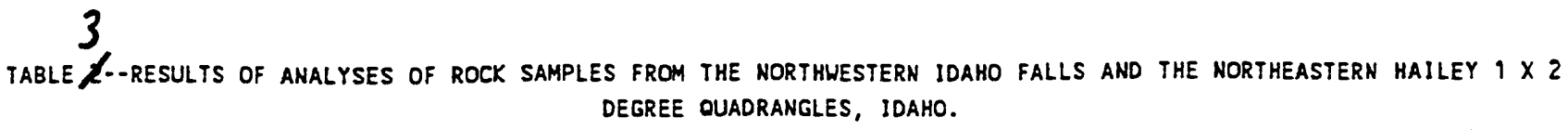

$[N$, not detected; <, detected but below the limit of determination shown; >, determined to be greater than the value shown.]

MACKAY QUADRANGLE

Sample Latitude Longitude Ca-pct. Fe-pet. Mg-pct. Na-pet.

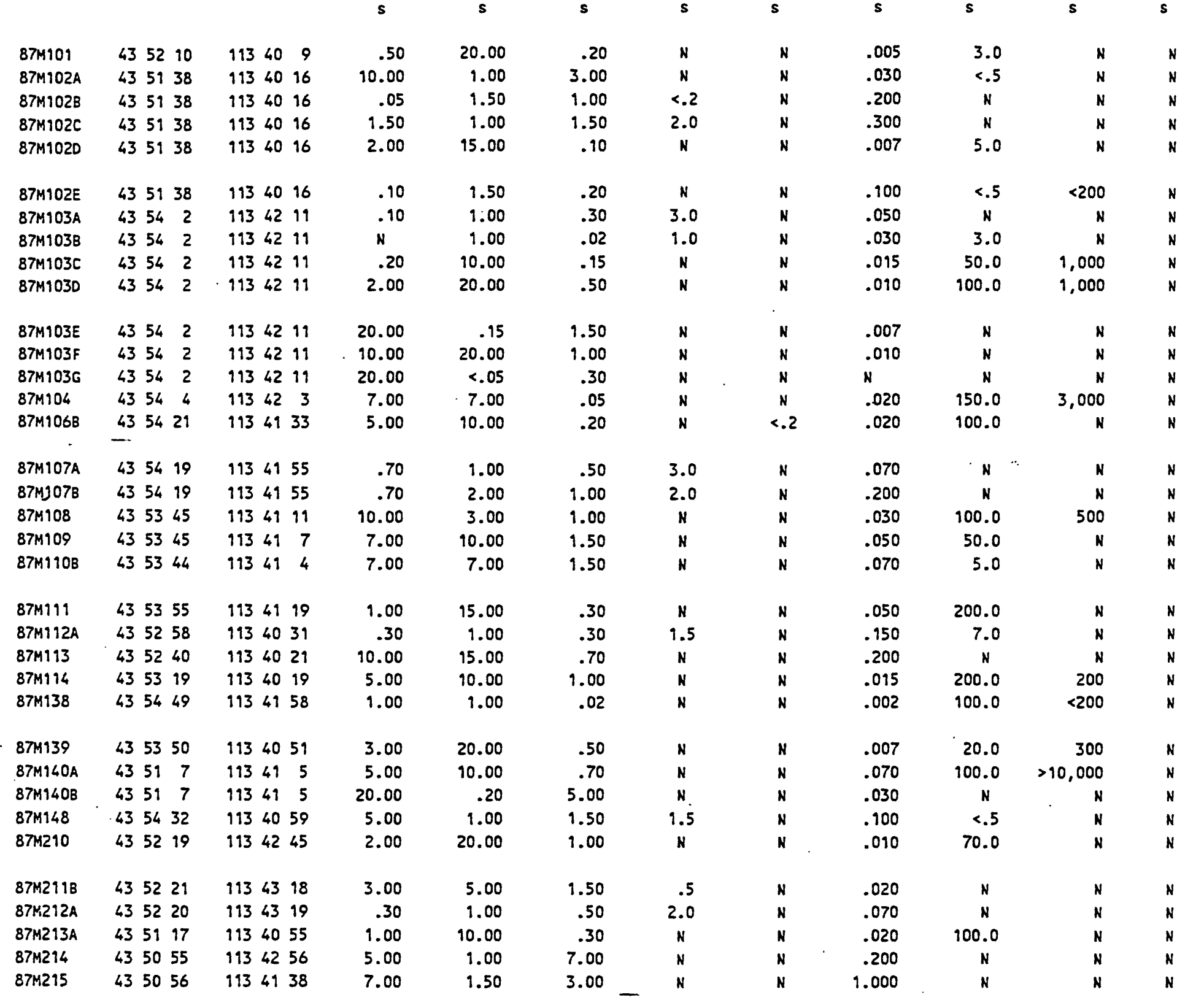

COPPER BASIN QUADRANGLE

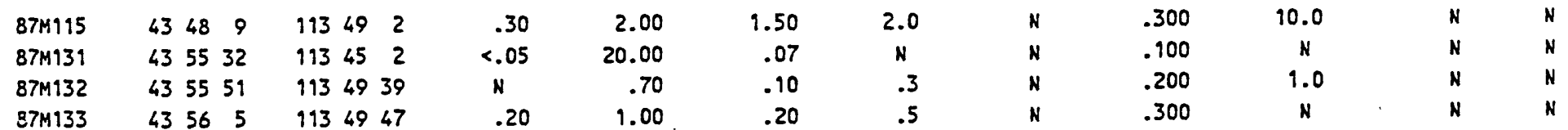


TABLE $k$--RESULTS OF ANALYSES OF ROCK SAMPLES FROM THE NORTHUESTERN IDAHO FALLS AND THE NORTHEASTERN HAILEY $1 \times 2$ DEGREE QUADRANGLES, IDAHO.---Continued

MACKAY QUADRANGLE

\begin{tabular}{|c|c|c|c|c|c|c|c|c|c|c|c|c|c|}
\hline Sample & $\begin{array}{c}\text { B-ppm } \\
\text { s }\end{array}$ & $\begin{array}{c}\text { Ba-ppm } \\
s\end{array}$ & $\begin{array}{c}\text { Be-ppn } \\
s\end{array}$ & $\begin{array}{c}\text { Bi-PPm } \\
s\end{array}$ & $\begin{array}{c}\text { cd-ppm } \\
s\end{array}$ & $\begin{array}{c}\text { Co-ppm } \\
s\end{array}$ & $\begin{array}{c}\text { Cr-ppm } \\
s\end{array}$ & $\begin{array}{c}\text { Cu-ppm } \\
s\end{array}$ & $\begin{array}{c}\text { Ga-ppm } \\
s\end{array}$ & $\begin{array}{c}\text { Ge-ppm } \\
\text { s }\end{array}$ & $\begin{array}{l}\text { La-ppm } \\
\quad s\end{array}$ & $\begin{array}{c}\text { Mn-ppm } \\
s\end{array}$ & $\begin{array}{c}\text { Mo-ppm } \\
\text { s }\end{array}$ \\
\hline $87 M 101$ & $\mathbf{N}$ & 20 & 7.0 & $N$ & N & 20 & $N$ & 1,000 & 50 & $N$ & $N$ & 2,000 & 20 \\
\hline $87 M 102 A$ & 15 & 50 & $N$ & $N$ & N & $N$ & 30 & 95 & 15 & $N$ & $N$ & 200 & $N$ \\
\hline $87 M 1028$ & 100 & 500 & 9.5 & $N$ & $N$ & $<10$ & 70 & 10 & 30 & $N$ & $N$ & 100 & $N$ \\
\hline $87 M 102 C$ & $<10$ & 1,000 & 9.0 & $N$ & $N$ & 95 & 50 & 95 & 20 & $N$ & 70 & 200 & $<5$ \\
\hline $87 M 1020$ & $<10$ & 70 & $N$ & 95 & 150 & $N$ & $<90$ & 500 & $<5$ & N & N & 5,000 & 30 \\
\hline 87M102E & 10 & 100 & N & $N$ & $N$ & $<10$ & 20 & 20 & 5 & $N$ & $N$ & 20 & 5 \\
\hline 87M103A & $<90$ & 200 & 2.0 & $N$ & $N$ & N & H & 7 & 50 & N & $<50$ & 950 & $<5$ \\
\hline $87 M 103 B$ & $N$ & 1,000 & $N$ & $N$ & N & $N$ & $N$ & 500 & 20 & N & $N$ & 10 & 10 \\
\hline $87 M 103 \mathrm{C}$ & $N$ & $<20$ & 9.5 & $N$ & $<20$ & 10 & $<90$ & 5,000 & 30 & $N$ & $N$ & 500 & 300 \\
\hline $87 M 103 D$ & N & 70 & 1.5 & 20 & $N$ & N & 10 & 2,000 & $<5$ & $N$ & $N$ & 1,500 & 15 \\
\hline 87M103E & $<10$ & 30 & $N$ & $N$ & N & $N$ & $<10$ & $<5$ & $\mathbf{N}$ & $N$ & $N$ & 70 & $<5$ \\
\hline $87 M 103 F$ & $N$ & $<20$ & 2.0 & N & N & $N$ & $N$ & 50 & 10 & $N$ & $N$ & 5,000 & $N$ \\
\hline $87 M 103 G$ & N & N & $N$ & N & N & $N$ & $N$ & $<5$ & $\mathbf{N}$ & $N$ & $N$ & 500 & N \\
\hline $87 M 104$ & $=<10$ & 150 & $N$ & 15 & N & $N$ & $N$ & 700 & 5 & $N$ & $N$ & 150 & 50 \\
\hline $87 M 1068$ & N & 3,000 & N & $N$ & N & $N$ & $<10$ & 70 & 15 & N & $N$ & 5,000 & 5 \\
\hline $87 M 107 A$ & 10 & 3,000 & N & $N$ & $N$ & $N$ & $N$ & 7 & 30 & $N$ & 150 & 100 & N \\
\hline $87 M 1078$ & 90 & 1,500 & $<1.0$ & $N$ & $N$ & $<10$ & 15 & 15 & 30 & $N$ & 50 & 300 & N \\
\hline $87 M 108$ & $<10$ & 70 & N & $<90$ & $N$ & $<10$ & 15 & 10,000 & $<5$ & $N$ & $N$ & 500 & $N$ \\
\hline $87 M 909$ & $<10$ & $N$ & N & $<90$ & 50 & 30 & 70 & 7,000 & 15 & $N$ & $N$ & 5,000 & 20 \\
\hline $87 M 190 B$ & $<10$ & $N$ & $N$ & N & $N$ & 10 & 100 & 7,000 & 10 & $N$ & $N$ & 1,000 & $N$ \\
\hline $87 M 911$ & $N$ & 500 & $N$ & 100 & 500 & 100 & N & 1,500 & 20 & $N$ & N & 700 & $N$ \\
\hline $87 M 912 A$ & N & 5,000 & N & N & N & $N$ & N & 700 & 30 & $N$ & $<50$ & 200 & 30 \\
\hline $87 M 113$ & $<90$ & N & N & $N$ & N & $<10$ & 20 & 70 & 20 & N & 50 & $>5,000$ & 50 \\
\hline $87 M 914$ & $<10$ & N & N & 70 & $<20$ & 70 & $<10$ & $>20,000$ & $N$ & $N$ & N & 500 & N \\
\hline $87 M 138$ & $<10$ & 30 & $N$ & $N$ & N & $N$ & $N$ & 300 & $\mathbf{N}$ & $N$ & N & 70 & N \\
\hline 87M139 & N & 70 & 2.0 & 50 & $<20$ & 10 & 10 & 10,000 & 30 & $N$ & $N$ & 1,500 & 7 \\
\hline $87 \mathrm{M} 140 \mathrm{~A}$ & $<10$ & 700 & $\mathbf{N}$ & 200 & N & 700 & 15 & 5,000 & 15 & $N$ & $N$ & 500 & 100. \\
\hline $87 M 140 B$ & 10 & 70 & N & $N$ & N & N & 70 & 5 & N & N & N & $<10$ & N \\
\hline $87 M 148$ & $N$ & 2,000 & $<1.0$ & $N$ & N & $<10$ & 20 & 30 & 20 & N & $<50$ & $150^{-}$ & 5 \\
\hline $87 M 210$ & $N$ & N & 1.5 & $<90$ & $N$ & 150 & 15 & $>20,000$ & 20 & $N$ & N & 1,000 & $<5$ \\
\hline 87M211B & $<10$ & 70 & 1.0 & N & $N$ & $<90$ & 20 & 30 & 30 & N & N & 2,000 & $<5$ \\
\hline 87M212A & N & 500 & 1.5 & $N$ & $N$ & N & $N$ & 15 & 50 & N & $<50$ & 150 & $N$ \\
\hline $87 M 213 A$ & $<10$ & 70 & 3.0 & 150 & 50 & 70 & N & 3,000 & 20 & $N$ & $N$ & $>5,000$ & 20 \\
\hline $87 M 214$ & 20 & 200 & N & N & $N$ & $N$ & 70 & N & $N$ & N & N & 150 & N \\
\hline 87M215 & 100 & 100 & $<1.0$ & 200 & N & N & 70 & $<90$ & 15 & $N$ & 100 & 300 & N \\
\hline
\end{tabular}

COPPER BASIN QUADRANGLE

\begin{tabular}{|c|c|c|c|c|c|c|c|c|c|c|c|c|c|}
\hline $87 M 915$ & 10 & 200 & $<1.0$ & 70 & N & $<90$ & 70 & 2,000 & 20 & $N$ & 70 & 30 & $N$ \\
\hline $87 M 131$ & $N$ & 1,000 & 1.0 & $N$ & N & N & 70 & 50 & 50 & $N$ & $N$ & $<10$ & N \\
\hline $87 M 132$ & $N$ & 1,000 & 3.0 & $N$ & $N$ & N & 30 & 5 & 30 & N & $<50$ & 15 & $N$ \\
\hline $87 \mathrm{M} 133$ & $N$ & 5.000 & $<9.0$ & N & N & N & $N$ & $<5$ & 30 & $N$ & $<50$ & 20 & $<5$ \\
\hline
\end{tabular}


TABLE 3 --RESULTS OF ANALYSES OF ROCK SAMPLES FROM THE NORTHWESTERN IDAHO FALLS AND THE NORTHEASTERN HAILEY $9 \times 2$ DEGREE QUADRANGLES, IDAHO.---CONTinUed

MACKAY QUADRANGLE

\begin{tabular}{|c|c|c|c|c|c|c|c|c|c|c|c|c|c|}
\hline Sample & $\begin{array}{c}\text { Nb-ppm } \\
s\end{array}$ & $\begin{array}{c}\mathrm{Ni}-\mathrm{ppm} \\
\mathrm{s}\end{array}$ & $\begin{array}{c}\text { Pb-ppn } \\
s\end{array}$ & $\begin{array}{c}\text { sb-ppm } \\
s\end{array}$ & $\begin{array}{c}\text { Sc-ppm } \\
s\end{array}$ & $\begin{array}{c}\text { Sn-ppm } \\
s\end{array}$ & $\begin{array}{c}\text { sr-ppm } \\
s\end{array}$ & $\begin{array}{c}\text { Th-ppm } \\
s\end{array}$ & $\begin{array}{c}V \cdot p p m \\
s\end{array}$ & $\begin{array}{c}W-p p m \\
s\end{array}$ & $\begin{array}{c}Y \text {-ppm } \\
s\end{array}$ & $\begin{array}{c}2 n-p p m \\
s\end{array}$ & $\begin{array}{c}2 r-p p m \\
s\end{array}$ \\
\hline $87 M 101$ & $<20$ & 5 & $N$ & $N$ & $N$ & 150 & $\mathbf{N}$ & $N$ & $<10$ & 1,000 & $<10$ & 1,000 & N \\
\hline $87 M 102 \mathrm{~A}$ & N & 20 & 20 & $N$ & $<5$ & $N$ & 150 & $N$ & 30 & $N$ & $<10$ & 500 & 15 \\
\hline $87 M 102 B$ & N & 7 & 10 & N & 5 & N & H & $N$ & 100 & N & N & N & 50 \\
\hline $87 M 102 C$ & $<20$ & 30 & 10 & N & 5 & $N$ & 150 & $N$ & 70 & N & $<10$ & $\mathbf{N}$ & 150 \\
\hline $87 M 1020$ & $N$ & 10 & 2,000 & N & $N$ & 15 & $N$ & $N$ & 15 & 150 & N & 7,000 & $N$ \\
\hline 87M102E & $N$ & 20 & 15 & $\mathbf{N}$ & $\mathbf{N}$ & N & N & N & 70 & 20 & N & $<200$ & 50 \\
\hline $87 M 103 \mathrm{~A}$ & 20 & $N$ & 70 & N & N & $N$ & N & $N$ & $<10$ & $N$ & N & N & 50 \\
\hline $87 M 103 B$ & $<20$ & $N$ & 9,000 & N & N & $N$ & $N$ & $N$ & N & N & N & 2,000 & 950 \\
\hline $87 M 103 C$ & N & 15 & 15,000 & $N$ & N & $N$ & N & $N$ & 50 & $<20$ & 20 & $>10,000$ & N \\
\hline 87M103D & N & 15 & 10,000 & $N$ & $N$ & 100 & $N$ & $N$ & 20 & 30 & $N$ & 10,000 & $N$ \\
\hline 87M103E & N & $N$ & 30 & N & $N$ & $N$ & 500 & $N$ & 30 & $N$ & $N$ & $N$ & N \\
\hline $87 M 103 F$ & N & $<5$ & 20 & $N$ & $N$ & 500 & $N$ & $N$ & $<10$ & 30 & N & 500 & N \\
\hline 87M103G & N & N & 15 & N & $N$ & N & 300 & $N$ & $<10$ & N & $N$ & N & N \\
\hline $87 M 104$ & $N$ & $N$ & $>20,000$ & $N$ & $N$ & 10 & 100 & N & 10 & 150 & N & 5,000 & $<10$ \\
\hline $87 M 906 B$ & N & 15 & $>20,000$ & N & $N$ & $N$ & 100 & N & 100 & N & N & 1,000 & $<10$ \\
\hline 87M107A & N & $N$ & 100 & N & $N$ & $N$ & 200 & N & 10 & $N$ & $<10$ & $N$ & 50 \\
\hline $87 M 107 B$ & $<20$ & 10 & 70 & N & $<5$ & N & 950 & N & 50 & N & N & N & 70 \\
\hline $87 M 108$ & N & 20 & 5,000 & N & N & 10 & 200 & N & 20 & $N$ & $<10$ & 1,000 & $<10$ \\
\hline $87 M 109$ & $N$ & 30 & 100 & N & $<5$ & 30 & $N$ & N & 20 & 20 & $<10$ & $>10,000$ & 10 \\
\hline $87 M 110 B$ & $N$ & 30 & 30 & N & $<5$ & 30 & $N$ & $N$ & 30 & $N$ & 15 & 3,000 & 15 \\
\hline $87 \times 111$ & $N$ & 20 & 15,000 & N & $N$ & $N$ & $\mathbf{N}$ & N & $<10$ & $N$ & $N$ & $>10,000$ & $<10$ \\
\hline $87 M 112 A$ & N & 7 & 100 & N & N & $\mathbf{N}$ & $<100$ & N & 15 & $<20$ & $N$ & 300 & 70 \\
\hline $87 M 113$ & $<20$ & 15 & 100 & N & $<5$ & 100 & N & N & 50 & $N$ & 10 & N & 70 \\
\hline $87 M 914$ & $N$ & 100 & $<10$ & N & $N$ & 70 & $N$ & $N$ & 10 & $<20$ & $N$ & 7,000 & $<10$ \\
\hline $87 M 138$ & N & 5 & 20,000 & 200 & $N$ & $N$ & $N$ & $N$ & 20 & $N$ & $N$ & 1,500 & N \\
\hline $87 M 939$ & N & 7 & $>20,000$ & N & $N$ & N & $N$ & $N$ & 10 & $N$ & $N$ & $>10,000$ & $N$ \\
\hline $87 M 140 A$ & N & 10 & 20,000 & $N$ & $N$ & $<10$ & N & N & 30 & 30 & $N$ & 3,000 & 50 \\
\hline $87 M 140 B$ & $N$ & 15 & 30 & $N$ & $N$ & $N$ & 300 & $N$ & 15 & $N$ & $<10$ & N. & 10 \\
\hline $87 M 148$ & N & 10 & 50 & $N$ & $<5$ & N & 300 & $N$ & 20 & N & $<10$ & 300 & 20 \\
\hline 87M210 & $N$ & 70 & 70 & N & $N$ & 200 & N & $N$ & $<10$ & 100 & N & 1,000 & $<10$ \\
\hline $87 M 211 B$ & N & 10 & 50 & $N$ & N & 100 & N & $N$ & 15 & $<20$ & $<10$ & $N$ & 95 \\
\hline $87 M 212 A$ & $<20$ & $<5$ & 70 & $N$ & $N$ & $<10$ & $N$ & $N$ & 10 & N & 10 & N & 50 \\
\hline 87M213A & N & 7 & $>20,000$ & $N$ & $N$ & $N$ & N & $N$ & 20 & 50 & $N$ & $>10,000$ & 10 \\
\hline $87 M 214$ & 50 & 30 & $<20$ & $N$ & N & N & N & $N$ & 50 & $<50$ & 70 & N & $>2,000$ \\
\hline $87 M 215$ & 100 & 20 & 20 & $N$ & $N$ & $<20$ & $N$ & $N$ & 100 & 100 & 100 & $N$ & $>2,000$ \\
\hline
\end{tabular}

\begin{tabular}{|c|c|c|c|c|c|c|c|c|c|c|c|c|c|}
\hline 8779115 & $<20$ & 30 & 10 & $N$ & 5 & $N$ & $N$ & $N$ & 50 & $N$ & N & $N$ & 70 \\
\hline $87 M 439$ & $N$ & $<5$ & 50 & N & $<5$ & $N$ & 150 & N & 50 & N & N & $N$ & 20 \\
\hline $87 M 132$ & $N$ & 5 & 30 & $N$ & $<5$ & N & $N$ & $N$ & 50 & $N$ & N & $N$ & 50 \\
\hline $87 M 133$ & $<20$ & N & 20 & $\kappa$ & $<5$ & $N$ & $N$ & $N$ & 20 & $N$ & $<10$ & $N$ & 150 \\
\hline
\end{tabular}


3
TAELE $/ 2$--RESULTS OF ANALYSES OF ROCK SAMPLES FROM THE NORTHUESTERN IDAHO FALLS AND THE NORTHEASTERN hAILEY $1 \times 2$ DEGREE QUADRANGLES, IDAHO.--Continued

MULDOON CANYON OUADRANGLE

\begin{tabular}{|c|c|c|c|c|c|c|c|c|c|c|c|}
\hline Sample & Latitude & Longitude & $\begin{array}{c}\text { Ca-pct. } \\
s\end{array}$ & $\begin{array}{c}\text { Fe-pct. } \\
s\end{array}$ & $\begin{array}{c}\text { Mg-pct. } \\
\text { s }\end{array}$ & $\begin{array}{c}\text { Na-pet. } \\
\text { s }\end{array}$ & $\begin{array}{c}\text { P-pct. } \\
s\end{array}$ & $\begin{array}{c}\text { Ti-pet. } \\
s\end{array}$ & $\begin{array}{c}A g-p p m \\
s\end{array}$ & $\begin{array}{c}\text { As - ppm } \\
s\end{array}$ & $\begin{array}{c}\text { Au-ppm } \\
s\end{array}$ \\
\hline 87M119A & $4342 \quad 29$ & 1935149 & 10.00 & 10.00 & 2.00 & N & N & .150 & 7.0 & N & N \\
\hline 87M119C & 434229 & 1935949 & .05 & 1.00 & 9.00 & .5 & N & .200 & $N$ & N & N \\
\hline $87 M 124 A$ & 433844 & 1134558 & $N$ & 1.00 & .50 & N & $N$ & .030 & $<.5$ & N & N \\
\hline $87 M 1248$ & 433844 & 1134558 & $<.05$ & 20.00 & .05 & N & $<.2$ & .030 & N & N & N \\
\hline $87 M 125$ & 434243 & 1135610 & 10.00 & 1.50 & 7.00 & .3 & $N$ & .050 & 2.0 & N & N \\
\hline $87 M 126$ & 434136 & 1135451 & .05 & 1.00 & .50 & 1.0 & N & .100 & .7 & $N$ & N \\
\hline $87 M 127$ & 434143 & 1135524 & 2.00 & 3.00 & 1.00 & $N$ & $N$ & .070 & 70.0 & N & N \\
\hline 87M128 & 434152 & 1135522 & $N$ & .10 & .03 & $N$ & N & .0 .15 & 30.0 & N & N \\
\hline $87 M 129$ & 434929 & 1135559 & 2.00 & 1.00 & 1.00 & 3.0 & N & .150 & N & N & N \\
\hline $87 M 130$ & $43 \quad 42 \quad 22$ & 1135620 & 5.00 & 3.00 & 9.00 & $N$ & N & .005 & 100.0 & N & N \\
\hline $87 M 216$ & 433921 & 1135652 & $<.05$ & .70 & .30 & .2 & N & .030 & $<.5$ & N & N \\
\hline $87 M 217$ & 43394 & 1135654 & 3.00 & 1.50 & 2.00 & N & N & .070 & .5 & N & N \\
\hline $87 M 218$ & 43399 & 9135633 & .70 & 2.00 & 1.50 & $\mathbf{N}$ & N & .070 & 2.0 & 5,000 & N \\
\hline $87 M 219$ & $4338 \quad 1$ & 1135629 & 2.00 & 3.00 & 2.00 & 2.0 & N & .300 & .5 & N & N \\
\hline $87 M 2208$ & 433754 & 9135648 & 1.00 & 2.00 & 2.00 & 1.0 & N & .200 & .7 & N & N \\
\hline $87 M 2218$ & 433754 & 1135639 & 2.00 & 2.00 & 2.00 & 1.0 & N & .150 & 1.0 & N & N \\
\hline
\end{tabular}

GROUSE QUADRANGLE

\begin{tabular}{|c|c|c|c|c|c|c|c|c|c|c|}
\hline $87 M 120$ & 43363 & 1133940 & 10.00 & 2.00 & .70 & .5 & $N$ & .030 & 9.5 & 9,500 \\
\hline $87 M 122$ & 433518 & $11340 \quad 7$ & $<.05$ & 9.50 & .50 & N & N & .200 & .7 & $N$ \\
\hline $87 \mathrm{M} 123$ & 433456 & $113 \quad 4024$ & $\mathbf{N}$ & .50 & .30 & N & N & .150 & 1.0 & N \\
\hline $87 M 134$ & 433549 & $113 \quad 3515$ & 1.50 & 5.00 & 1.50 & N & N & .300 & 15.0 & N \\
\hline $87 M 135$ & 43363 & 1133420 & $\mathrm{~N}$ & .50 & $<.02$ & N & N & .200 & 70.0 & N \\
\hline 87 M136 & 433557 & 11334 & $<.05$ & 5.00 & .02 & N & $<.2$ & .200 & 30.0 & 200 \\
\hline
\end{tabular}

PHI KAPPA MOUNTAIN QUADRANGLE

\begin{tabular}{|c|c|c|c|c|c|c|c|c|c|c|}
\hline $87 M 143$ & $4350 \quad 48$ & 1141210 & 5.00 & 5.00 & 5.00 & N & N & .100 & 100.0 & N \\
\hline $87 M 199$ & $43 \quad 49 \quad 18$ & $114 \quad 1019$ & $<.05$ & 2.00 & .30 & N & N & .070 & 10.0 & N \\
\hline $87 M 207 A$ & 435128 & 114146 & $N$ & .70 & $<.02$ & N & N & .003 & 1.0 & 1,000 \\
\hline $87 M 207 C$ & 435128 & 19414 & .05 & 9.00 & 1.00 & .7 & $N$ & .150 & 5.0 & N \\
\hline 87M208A & 435213 & 1141434 & 2.00 & 1.50 & 1.50 & N & $<.2$ & .950 & 2.0 & 200 \\
\hline $87 M 209$ & $4359 \quad 2$ & 9149456 & 5.00 & 5.00 & 1.50 & .7 & .2 & .030 & $N$ & $N$ \\
\hline
\end{tabular}


tABLE 2 -resesults of ANALYSES OF ROCK SAMPLES FROM THE NORTHWESTERN IDAHO FALLS AND THE NORTHEASTERN HAILEY $1 \times 2$ DEGREE QUADRANGLES, IDAHO.---CONTinUEd

MULDOON CANYON QUADRANGLE

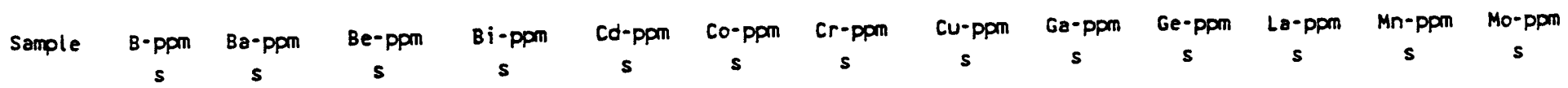

\begin{tabular}{|c|c|c|c|c|c|c|c|c|c|c|c|c|c|}
\hline $87 M 119 \mathrm{~A}$ & $<10$ & 50 & 1.0 & $N$ & $<20$ & 10 & 30 & 1,000 & 30 & $N$ & N & 2,000 & $<5$ \\
\hline $87 M 119 C$ & 30 & 3,000 & $<1.0$ & N & N & $<10$ & 30 & 50 & 10 & $N$ & $N$ & 150 & N \\
\hline $87 M 124 A$ & 15 & 500 & $N$ & N & N & $N$ & 10 & 15 & $N$ & $N$ & N & 50 & $N$ \\
\hline $87 M 124 B$ & $N$ & 100 & 1.0 & N & $N$ & N & $N$ & 50 & 30 & $N$ & N & 10 & N \\
\hline $87 M 125$ & $N$ & 3,000 & $N$ & $<10$ & $N$ & N & 20 & 100 & 15 & $N$ & $N$ & 500 & $N$ \\
\hline $87 M 126$ & $<10$ & 500 & $<1.0$ & N & $N$ & $<10$ & 30 & 10 & 20 & $N$ & N & 1,000 & $<5$ \\
\hline $87 M 127$ & $<10$ & 700 & 2.0 & 100 & 50 & 10 & 20 & 15,000 & 30 & $N$ & $N$ & 5,000 & 100 \\
\hline $87 M 128$ & $N$ & 700 & $N$ & 100 & N & N & N & 500 & N & N & $N$ & $<10$ & 20 \\
\hline $87 M 129$ & 20 & 1,500 & $<1.0$ & $N$ & $N$ & N & 50 & 20 & 70 & N & $N$ & 150 & $N$ \\
\hline $87 M 130$ & $N$ & 100 & 1.5 & 70 & 500 & $<10$ & N & 7,000 & 5 & N & $<50$ & 3,000 & $<5$ \\
\hline $87 M 216$ & 15 & 2,000 & 9.0 & $N$ & N & $N$ & N & 50 & 30 & N & N & 50 & $N$ \\
\hline $87 M 217$ & 30 & 1,500 & $<1.0$ & N & N & $N$ & 20 & 30 & 5 & N & N & 200 & $N$ \\
\hline $87 M 218$ & -70 & 300 & 5.0 & N & $N$ & $<10$ & 30 & 50 & 15 & N & $N$ & 300 & 15 \\
\hline $87 M 219$ & $<10$ & 2,000 & $<9.0$ & N & N & 20 & 300 & 30 & 30 & N & 50 & 700 & $N$ \\
\hline $87 M 220 B$ & 10 & 1,000 & $<1.0$ & N & N & 15 & 200 & 50 & 20 & N & $<50$ & 500 & $N$ \\
\hline $87 M 2218$ & 15 & 2,000 & $<1.0$ & $N$ & $N$ & 15 & 200 & 30 & 30 & N & 50 & 1,000 & N \\
\hline
\end{tabular}

\begin{tabular}{|c|c|c|c|c|c|c|c|c|c|c|c|c|c|}
\hline $87 M 120$ & $<10$ & 200 & $N$ & $N$ & $N$ & $N$ & 10 & 15 & $<5$ & $N$ & $N$ & 300 & $N$ \\
\hline $87 M 122$ & 70 & 2,000 & $<1.0$ & $N$ & $N$ & $N$ & 70 & 30 & 5 & $N$ & $N$ & $N$ & $N$ \\
\hline $87 M 123$ & 30 & 700 & $N$ & $N$ & $N$ & $N$ & 20 & 10 & 5 & $N$ & N & 95 & $\mathbf{N}$ \\
\hline $87 M 134$ & 10 & 500 & $N$ & $<10$ & $N$ & 15 & 200 & 100 & 20 & $N$ & $<50$ & 700 & $<5$ \\
\hline $87 M 135$ & $N$ & 50 & $N$ & $N$ & $N$ & $N$ & 10 & 20 & 30 & $N$ & $N$ & N & $N$ \\
\hline 87M136 & $<10 \ldots$ & _. 700 & $-N$ & $N$ & $N$ & $N$ & 20 & 150 & 70 & $N$ & 50 & 10 & 10 \\
\hline
\end{tabular}

\section{PHI KAPPA MOUNTAIN QUADRANGLE}

\begin{tabular}{|c|c|c|c|c|c|c|c|c|c|c|c|c|c|}
\hline $87 M 143$ & $<10$ & 100 & $<1.0$ & 100 & 20 & 10 & 30 & 50 & $N$ & $N$ & N & 5,000 & $<5$ \\
\hline $87 M 199$ & 10 & 200 & $N$ & 10 & $N$ & $N$ & 10 & 50 & $<5$ & N & N & 300 & 5 \\
\hline $87 M 207 A$ & $N$ & 20 & $N$ & 30 & N & $N$ & $N$ & 50 & $N$ & N & N & $<10$ & 20 \\
\hline $87 M 207 C$ & 10 & 1,500 & 5.0 & 15 & $N$ & N & 30 & 70 & 30 & N & $<50$ & 70 & $<5$ \\
\hline 87M208A & $<10$ & 300 & 2.0 & $N$ & $<20$ & 15 & 70 & 50 & 5 & $N$ & $N$ & 700 & 100 \\
\hline 87M209 & $<10$ & 200 & 5.0 & $N$ & $N$ & $<10$ & 30 & 100 & $N$ & 20 & 100 & $>5,000$ & 10 \\
\hline
\end{tabular}


TABLE $\{$--RESULTS OF ANALYSES OF ROCK SAMPLES FROM THE NORTHWESTERN IDAHO FALLS AND THE NORTHEASTERN HAILEY $1 \times 2$ DEGREE QUADRANGLES, IDAHO.---CONTinUed

MULDOON CANYON QUADRANGLE

\begin{tabular}{|c|c|c|c|c|c|c|c|c|c|c|c|c|c|}
\hline Sample & $\begin{array}{l}\text { Nb-ppm } \\
\text { s }\end{array}$ & $\begin{array}{c}\text { Ni-ppm } \\
\text { s }\end{array}$ & $\begin{array}{l}\text { Pb-ppm } \\
s\end{array}$ & $\begin{array}{c}\text { sb-ppm } \\
s\end{array}$ & $\begin{array}{c}\text { Sc-ppm } \\
\text { s }\end{array}$ & $\begin{array}{c}\text { Sn-ppm } \\
s\end{array}$ & $\begin{array}{c}\text { Sr-ppm } \\
\text { s }\end{array}$ & $\begin{array}{c}\text { Th-ppm } \\
s\end{array}$ & $\begin{array}{c}V-p p m \\
s\end{array}$ & $\begin{array}{c}W-p p m \\
s\end{array}$ & $\begin{array}{c}Y-p p m \\
s\end{array}$ & $\begin{array}{c}2 n-p p m \\
s\end{array}$ & $\begin{array}{c}\text { Zr-ppm } \\
s\end{array}$ \\
\hline 87MI19A & N & 95 & 2,000 & N & 5 & $<10$ & 300 & N & 150 & $N$ & $<10$ & 10,000 & 50 \\
\hline $87 M 119 \mathrm{C}$ & N & 15 & 10 & N & $<5$ & N & N & N & 150 & N & N & $<200$ & 50 \\
\hline 87Mi24A & N & 15 & N & N & N & N & $N$ & N & 70 & N & $N$ & N & 30 \\
\hline $87 M 124 B$ & N & 10 & $N$ & N & N & N & N & N & 150 & N & $N$ & 500 & 20 \\
\hline $87 \mathrm{M} 125$ & N & 10 & 100 & N & $<5$ & N & 900 & N & 50 & N & N & N & 10 \\
\hline $87 \mathrm{M} 126$ & N & 20 & 200 & N & $N$ & N & N & N & 100 & N & N & 300 & 50 \\
\hline $87 \times 127$ & $\mathbf{N}$ & 50 & 20,000 & N & $<5$ & 30 & 200 & N & 150. & 30 & $<10$ & $>10,000$ & 30 \\
\hline $87 M 128$ & N & N & 1,000 & N & N & N & N & N & 20 & N & N & 1,500 & $<10$ \\
\hline $87 M 129$ & N & 5 & 50 & N & $<5$ & N & $<100$ & N & 70 & $N$ & $N$ & $N$ & 30 \\
\hline $87 M 130$ & N & N & $>20,000$ & N & H & 20 & 200 & N & 30 & $<20$ & 90 & $>10,000$ & $\mathrm{~N}$ \\
\hline 874216 & N & 7 & 50 & N & N & N & N & N & $<90$ & $N$ & $\mathbf{N}$ & N & 20 \\
\hline $87 M 217$ & N & 15 & 150 & N & $<5$ & N & N & N & 100 & N & $<10$ & 200 & 20 \\
\hline $87 M 218$ & N & 70 & 50 & $N$ & $<5$ & N & $N$ & N & 200 & N & $\mathbf{N}$ & 500 & 20 \\
\hline 87M219 & $<20$ & 50 & 20 & N & 7 & N & 100 & N & 70 & N & $<10$ & $N$ & 70 \\
\hline 37M220B & N & 50 & 20 & N & 5 & $N-$ & $<100$ & N & 50 & N & $<10$ & N & 50 \\
\hline 8712218 & N & 50 & 50 & N & 5 & N & 100 & $N$ & 70 & $N$ & $<10$ & $\mathbf{N}$ & 70 \\
\hline
\end{tabular}

GROUSE QUADRANGLE

\begin{tabular}{|c|c|c|c|c|c|c|c|c|c|c|c|c|c|}
\hline $87 M 120$ & $N$ & 5 & 150 & N & N & $N$ & 200 & N & 20 & $N$ & $N$ & 200 & 10 \\
\hline $87 M 122$ & N & 10 & N & $N$ & $<5$ & $\mathbf{N}$ & N & $N$ & 200 & $N$ & $N$ & $N$ & 50 \\
\hline $87 M 123$ & $<20$ & 5 & 20 & $\mathbf{N}$ & N & $N$ & $N$ & $N$ & 150 & $N$ & $N$ & N & 70 \\
\hline $87 M 134$ & N & 30 & 100 & $N$ & 5 & N & $<100$ & $N$ & 100 & N & $<10$ & $<200$ & 70 \\
\hline 87M135 & $N$ & $<5$. & 500 & N & $<5$ & N & 150 & $N$ & 50 & $N$ & N & N & 70 \\
\hline $87 m 136$ & N & $<5$ & 700 & N & 5 & H & 1,000 & $N$ & 200 & N & N & $\mathbf{N}$ & 70 \\
\hline
\end{tabular}

PHI KAPPA MOUNTAIN QUADRANGLE

\begin{tabular}{|c|c|c|c|c|c|c|c|c|c|c|c|c|c|}
\hline $87 M 143$ & $N$ & 20 & 5,000 & N & $<5$ & 30 & N & N & 70 & $N$ & $<10$ & 2,000 & 50 \\
\hline $87 M 199$ & N & 10 & 1,000 & N & N & 10 & N & N & 70 & $N$ & $N$ & $<200$ & 50 \\
\hline 87M207A & N & $<5$ & 10 & $N$ & $N$ & N & N & N & 95 & 300 & N & N & N \\
\hline $87 M 207 C$ & N & N & 9,000 & N & $<5$ & 70 & N & N & 50 & $<20$ & N & N & 70 \\
\hline 87M208A & N & 300 & 200 & N & 5 & $<10$ & $N$ & N & 9,500 & N & 15 & 1,000 & 30 \\
\hline $87 M 209$ & N & 50 & $<10$ & N & $<5$ & $\mathbf{N}$ & N & N & 200 & 30 & 10 & 300 & 10 \\
\hline
\end{tabular}

